Document downloaded from:

http://hdl.handle.net/10251/97734

This paper must be cited as:

Cerdeira, M.; Kurlyandskaya, G.; Fernandez, A.; Tejedor, M.; García Miquel, AH. (2003). Giant magnetoimpedance effect in surface modified CoFeMoSiB amorphous ribbons. Chinese Physics Letters. 20(12):2246-2249. doi:10.1088/0256-307X/20/12/045

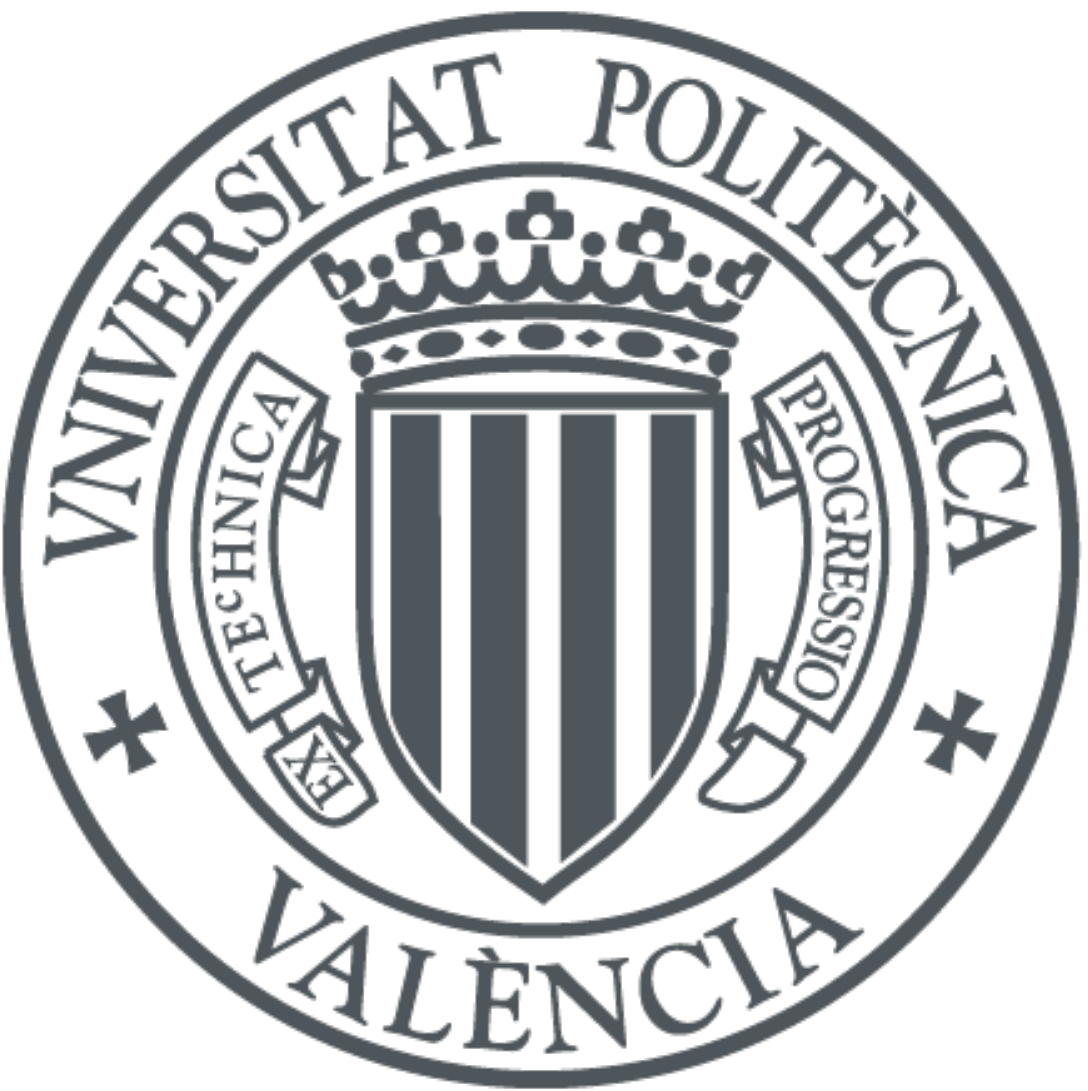

The final publication is available at

https://doi.org/10.1088/0256-307X/20/12/045

Copyright IOP Publishing

Additional Information 


\title{
Giant Magnetoimpedance Effect in Surface Modified CoFeMoSiB Amorphous Ribbons *
}

\author{
M. A. Cerdeira ${ }^{1}$, G. V. Kurlyandskaya ${ }^{1}$, A. Fernandez ${ }^{1}$, M. Tejedor ${ }^{1}$, H. Garcia-Miquel $^{2}$ \\ ${ }^{1}$ Universidad de Oviedo, Facultad de Ciencias, Departamento de Fisica, Avda Calvo Sotelo s/n, 33007 Oviedo, \\ Asturias, Spain \\ ${ }^{2}$ Grupo de Sensores y Magnetismo, Departamento de Ingeniería Electrónica, Universidad Politécnica de Valencia, \\ Camino de Vera s/n, 46021 Valencia, Spain
}

Thin magnetic Fe layers in thickness of 10-240 nm were deposited onto a wheel surface of CoFeMoSiB amorphous ribbons to check our concept of a new type of heterogeneous magnetoimpedance materials formed by two different magnetic parts. The presence of an additional iron layer modifies the magnetoimpedance response of the composite material and leads to increase of the magnetoimpedance ratio from 330 to $345 \%$ at a frequency of $3.5 \mathrm{MHz}$. Two possible mechanisms are discussed for explanation to the observed behaviour. Modification of the surface properties of the amorphous ribbons may have certain potential for technological applications.

Appropriate soft magnetic materials can show large variation of the total impedance upon application of the external magnetic field. This phenomenon, called the magnetoimpedance (MI) effect, attracts special attention due to the possibility of being employed in many technological applications based on the sensing of very small magnetic field. ${ }^{[1,2]}$ Magnetoimpedance effect was observed in amorphous and nanocrystalline ribbons, ${ }^{[3,4]}$ wires, ${ }^{[1]}$ thin films and sandwiches, ${ }^{[5-7]}$ electroplated wires, ${ }^{[8]}$ microwires ${ }^{[9]}$ ribbon/wire ${ }^{[10]}$ and ribbon/ribbon ${ }^{[11]}$ composites. There are heterogeneous MI materials mentioned above: three-layered sandwiches, electroplated wires and composites. The heterogeneous structures are formed by non-magnetic conductive core and a magnetic external part. The advantages of these materials to some extent are based on the difference of conductivities of the external and internal parts, i.e., the $\sigma_{1} / \sigma_{2}$ ratio, of the magnetic and non-magnetic layers. The theory of the magnetoimpedance for ferromagnet/non-magnetic conductor/ferromagnet structures was written recently and many experimental results were reasonably well described. ${ }^{[12,13]}$ We have developed a concept of another type of heterogeneous MI material, which has two different magnetic layers. It is based on the well known ${ }^{[14]}$ dependence of skin penetration depth $\delta$ on transverse or circular magnetic permeability of ferromagnet: $\delta=\left[2 /\left(\omega \mu_{0} \mu \sigma\right)\right]^{1 / 2}$, where $\omega$ is an alternating current angular frequency, $\mu_{0}$ is the vacuum permeability, and $\mu$ is the effective magnetic permeability. Effective magnetic permeability can be changed by thermal treatments in a magnetic field or stress annealing. ${ }^{[3,4,15]}$ These treatments affect the whole sample. It is known that in certain circumstances, amorphous or nano-crystalline magnetic materials can show non-uniform anisotropy, i.e. different properties, for example, near the surface or in the volume of the ribbon. ${ }^{[3,16,17]}$ Frequently, it is difficult to avoid this because of the fabrication method. Let us define the ribbon surface which has a contact with a wheel during fabrication as the wheel surface and the opposite one as an air surface. The surface roughness of the air and the roughness of wheel surfaces of the ribbons rapidly quenched by a single roller technique are different from each other due to air pockets on the wheel surface. ${ }^{[15]}$ Magnetoimpedance response, being very sensitive to the features especially near the surface characteristics, reflects them and, we suppose, even can be improved by appropriate surface modification. Significant changes of the anisotropy and magnetic properties were reported recently for the heterogeneous samples fabricated by radio frequency sputtering deposition of an additional $\mathrm{FeNi}$ magnetic layer onto the surface of the $\mathrm{FeCoNi} / \mathrm{CuBe}$ electroplated wire. ${ }^{[15]}$ Therefore we propose the deposition of thin magnetic layer as a method of modification of surface properties and magnetoimpedance response. In this Letter, we describe our experience in fabrication and study of surface modified $\mathrm{CoFeMoSiB}$ amorphous ribbons for magnetoimpedance applications.

We have selected single-roller quenched $\mathrm{Co}_{67} \mathrm{Fe}_{4} \mathrm{Mo}_{1.5} \mathrm{Si}_{16.5} \mathrm{~B}_{11}$ amorphous ribbons as a basic material because they show very high MI effect for relatively low driving current intensity, low saturation magnetostriction of $2.8 \times 10^{-7[4,18]}$ and therefore 
they can be considered as a model material. The as-quenched ribbons were annealed at $340^{\circ} \mathrm{C}$ for one hour for homogenization and cut on $1 \times 0.02 \times 100 \mathrm{~mm}$ samples prior to any additional deposition. Afterwards very thin pure iron films were deposited by evaporation in a vacuum of $10^{-3}$ bar on the wheel surface of the amorphous ribbon. The aim was to decrease the roughness of the surface and domain walls pinning, but not to increase the coercivity, by the decay of the nucleation process as it happens if we polish the surface. ${ }^{[16]}$ One may suppose the exchange interaction in the interface part between thin Fe layer and $\mathrm{CoFeMoSiB}$ basis. At the same time as we deposited Fe layer onto the ribbon, the same layer was deposited onto a glass substrate in order to control self properties of the Fe layer. Although we realize that the structural state, anisotropy and magnetic properties of the Fe layer deposited onto a glass substrate are not the same as properties of the layer deposited onto the amorphous ribbon, it is useful to study the Fe layer as a reference. The surfaces of all the samples were examined by a JEOL $60 \mathrm{kV}$ scanning electron microscope (SEM). The longitudinal hysteresis loops of amorphous $\mathrm{CoFeMoSiB}$ ribbons were measured by an inductive method for frequency of $100 \mathrm{~Hz} .{ }^{[14]}$ The magneto optic transverse Kerr effect (MOKE) was used for the measurements of hysteresis loops of thin Fe films. Some measurements of saturation magnetization and perpendicular anisotropy of Fe films were carried out by the Miyajima ${ }^{[19]}$ method. Magnetoimpedance was measured by a four-point technique using an imprinted circuit for the frequency of $3.5 \mathrm{MHz}$ and sinusoidal driving current intensity of $I_{\mathrm{rms}}=3 \mathrm{~mA}$ flowing through the sample along the ribbon axis. A bias magnetic field up to $12000 \mathrm{~A} / \mathrm{m}$ was applied parallel to the ribbon axis. The MI ratio was defined as follows:

$$
\Delta Z / Z(H)=\frac{\left[Z(H)-Z\left(H_{12000 \mathrm{~A} / \mathrm{m}}\right)\right]}{Z\left(H_{12000 \mathrm{~A} / \mathrm{m}}\right)} \times 100 \% .
$$

The inset of Fig. 1 shows the hysteresis loop of the amorphous ribbon without any additional deposited layer. The effective magnetic anisotropy is a longitudinal one. At the same time through the doublepeak shape of the MI response in a very small field [Fig. 1(a)], one may see that there is a weak nonlongitudinal component. A similar situation was discussed earlier for nano-crystalline FINEMET ribbons where a very weak anisotropy with a transverse component was detected just after nanocrystallization without any additional treatment for very soft material with longitudinal effective anisotropy. ${ }^{[4]}$ Perhaps partially this non-longitudinal component appears due to surface anisotropy features. Another reason to detect a non-longitudinal component could be the presence of well-known high order anisotropy. ${ }^{[20,21]}$
The total value of the magnetoimpedance ratio of the $\mathrm{CoFeMoSiB}$ ribbon without surface modification is rather high at about $330 \%$. The deposition of a $10 \mathrm{~nm}$ Fe layer in the case of consideration leads to the increases of both the MI ratio and MI sensitivity in a very small field of $\pm 180 \mathrm{~A} / \mathrm{m}$ for about $15 \%$. Further increase of the Fe layer thickness up to $500 \AA$ and more (Fig. 1(b)) leads to decay of the MI response down to the values less than the ones corresponding to the amorphous ribbon with no $\mathrm{Fe}$ layer deposition. Why does the MI change? Why is the MI value higher for the composite material of $\mathrm{Co}_{67} \mathrm{Fe}_{4} \mathrm{Mo}_{1.5} \mathrm{Si}_{16.5} \mathrm{~B}_{11}$ amorphous ribbon plus $10 \mathrm{~nm}$ Fe film? We suppose that two mechanisms could contribute in this phenomenon. The first one is an exchange sub-magnetization of the wheel surface of the amorphous ribbon due to the presence of the thin Fe layer. The second one is based on the abovementioned fact ${ }^{[3,16,17]}$ of possible non-uniformity of the anisotropy of the amorphous ribbons.
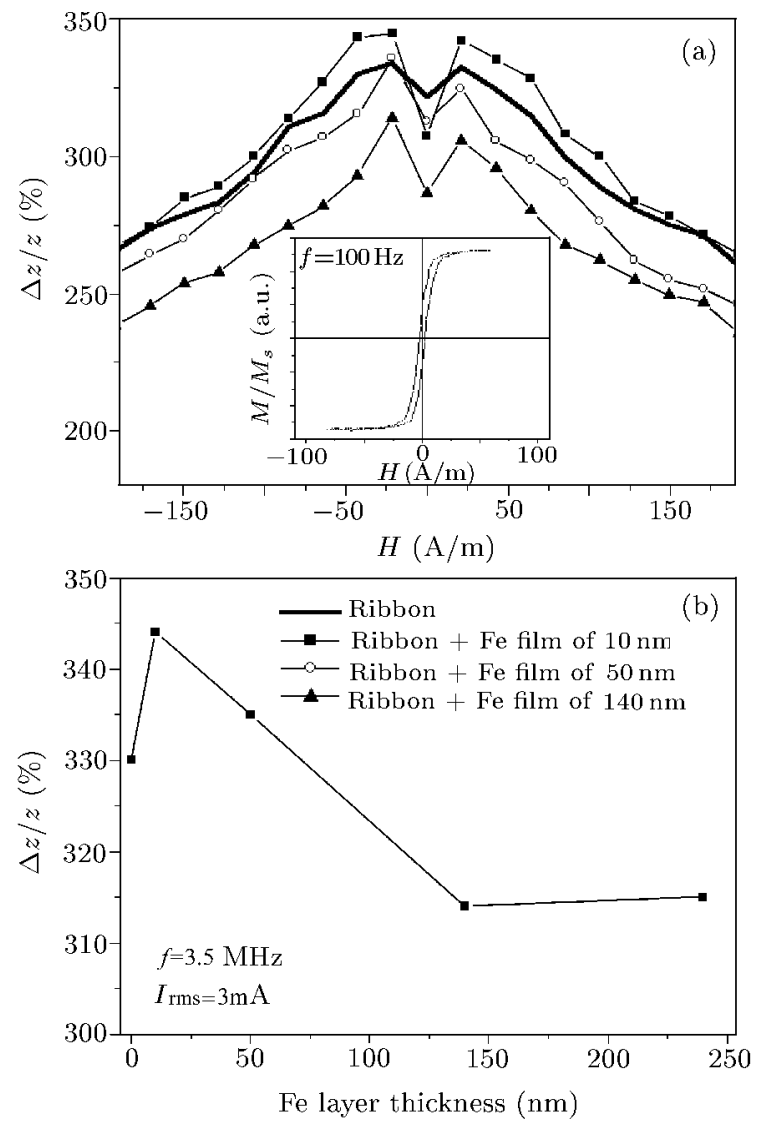

Fig. 1. (a) MI responses of $\mathrm{Co}_{67} \mathrm{Fe}_{4} \mathrm{Mo}_{1.5} \mathrm{Si}_{16.5} \mathrm{~B}_{11}$ amorphous ribbon with additional pure Fe layer of different thickness deposited onto wheel surface of the ribbon; (b) maximum of the MI ratio as a function of the thickness of Fe layer deposited onto wheel surface of the ribbon. Inset: Longitudinal hysteresis loop of $\mathrm{Co}_{67} \mathrm{Fe}_{4} \mathrm{Mo}_{1.5} \mathrm{Si}_{16.5} \mathrm{~B}_{11}$ amorphous ribbon with no additional layers.

Although the structural state, anisotropy, and magnetic properties of the iron layer deposited onto 
a glass substrate are not exactly the same as of the properties of the layer deposited onto the $\mathrm{CoFeMoSiB}$ ribbon, some useful information can be taken from the study of the properties of the iron layers deposited onto the glass substrate.

Figure 2 shows the hysteresis loops of the Fe films deposited onto glass substrates. The films have the same thickness as deposited onto $\mathrm{Co}_{67} \mathrm{Fe}_{4} \mathrm{Mo}_{1.5} \mathrm{Si}_{16.5} \mathrm{~B}_{11}$ amorphous ribbons for MI measurements. It is seen that an increase of the film thickness results in growth of the coercivity and change of the shape of the hysteresis loop. Rather square hysteresis loop corresponds to the $10 \mathrm{~nm}$ film with saturation for $3000 \mathrm{~A} / \mathrm{m}$ field, the highest value of the MOKE intensity and most probably in-plane effective magnetization. Although the details of this study will be reported elsewhere ${ }^{[22]}$ one may mention that a simple analysis of torque of Fe films by the Miyajima method ${ }^{[19]}$ shows that all the films have a perpendicular magnetic anisotropy component which is very high for thin films, high for thick films, and has a minimum value for the thickness of about $100 \mathrm{~nm}$.

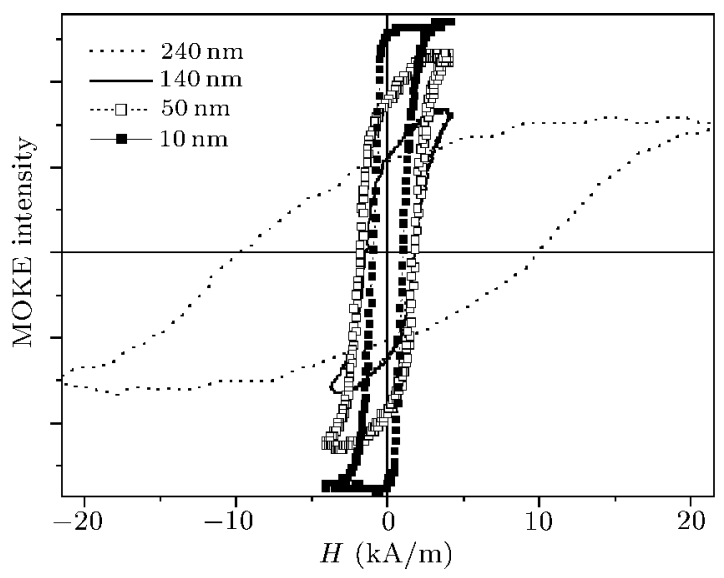

Fig. 2. MOKE hysteresis loops of Fe thin films of different thickness deposited onto a glass substrate by the thermal evaporation method.

Figure 3 shows the MOKE intensity of the $10 \mathrm{~nm}$ Fe film and MI responses of the ribbon without deposition, and with the $10 \mathrm{~nm}$ Fe layer deposited onto the wheel surface. The sub-magnetization of the ribbon wheel surface by the Fe film decays very fast towards the centre of the ribbon and therefore has a significant influence in the interface zone between the film and the ribbon. Two vertical dashed lines in Fig. 3 indicate the field range of almost constant MOKE signal intensity. The same as a permanent magnet in the multilayered structures creates the biased field, the Fe layer influences on the amorphous ribbon surface. The superposition of the external field and a field created by the Fe thin layer defines the effective field near the surface of the ribbon. For the $10 \mathrm{~nm}$-thick Fe film this modification is favourable to increase of the transverse permeability and MI. When the thickness of the iron layer increases, the Fe layer anisotropy changes and MI decays.

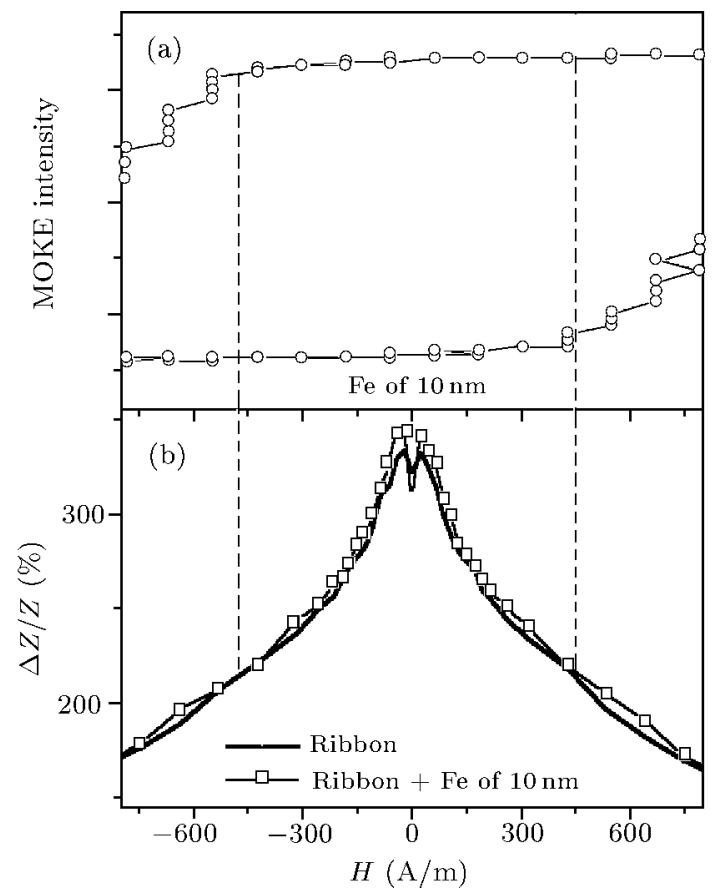

Fig. 3. MOKE hysteresis loop of the $10 \mathrm{~nm}$ Fe thin film deposited onto a glass substrate (a) and MI responses of $\mathrm{Co}_{67} \mathrm{Fe}_{4} \mathrm{Mo}_{1.5} \mathrm{Si}_{16.5} \mathrm{~B}_{11}$ amorphous ribbon with additional $10 \mathrm{~nm}$ Fe layer and without it (b). MI measurements is carried out for ac current frequency of $3.5 \mathrm{MHz}$ and intensity of $3 \mathrm{~mA}$. The dashed vertical lines indicate the area where the non-modified sample shows lower MI response.

The second possible explanation is based on the above-mentioned fact ${ }^{[3,16,17]}$ of possible nonuniformity of the anisotropy of the amorphous ribbons. Figure 4 shows the morphology of sample surface before and after the Fe-layer deposition. The deposition of the iron layer results in a modification of the roughness of the surface by a decrease of the depth of the Fe pockets. In order to obtain more information with respect to surface roughness changes the $\mathrm{x}$-ray reflectivity techniques or spin tunnelling microscopy can be proposed. Also, more complex model considering the effect of surface and the interface roughness can be proposed for the development in the future. The deposition of the iron layer does not impede the nucleation of the domain process like in the case of polishing the ribbon surface ${ }^{[16]}$ but perhaps makes it easier for the domain wall displacements because of more favourable magnetostatic balance. One may suppose that the deposition of the additional layer, made of the same composition by rf-sputtering or by evaporation methods, could be the most appropriate technological procedure if the proposed mechanism really takes place. Therefore it could be interesting to study the 
influence of the deposition of a thin layer of the same $\mathrm{Co}_{67} \mathrm{Fe}_{4} \mathrm{Mo}_{1.5} \mathrm{Si}_{16.5} \mathrm{~B}_{11}$ composition in the future. It could be useful not only for magnetoimpedance applications but for all cases when as uniform as possible anisotropy is desired.
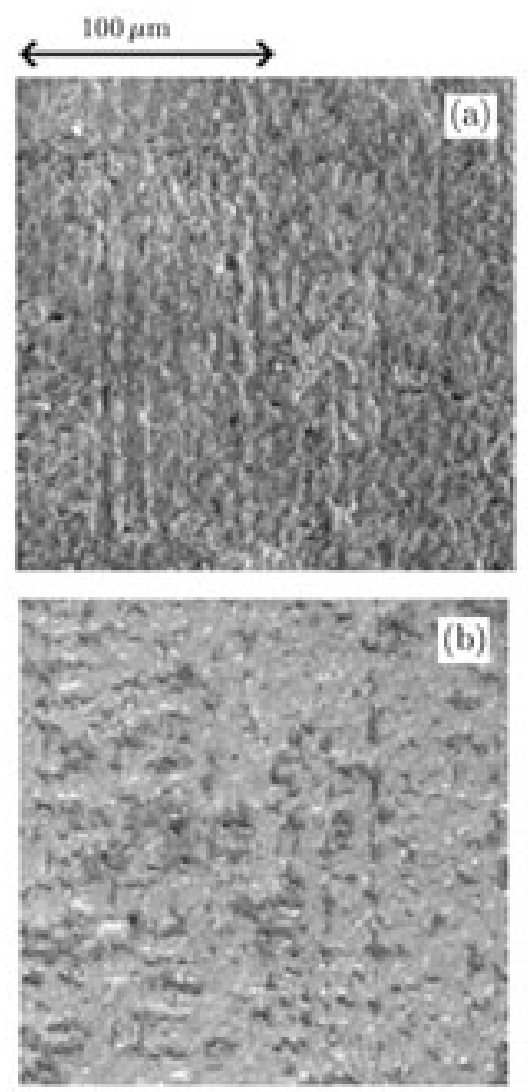

Fig. 4. SEM images of the wheel $\mathrm{Co}_{67} \mathrm{Fe}_{4} \mathrm{Mo}_{1.5} \mathrm{Si}_{16.5} \mathrm{~B}_{11}$ amorphous surface: (a) without any surface modification; (b) surface modified by evaporation deposition of additional Fe layer of $10 \mathrm{~nm}$.

In summary, we have reported the results of the study of magnetoimpedance effect in CoFeMoSiB amorphous ribbons with a surface, modified by a deposition of an additional Fe layer. The magnetoimpedance value depends on the thickness of the iron layer. Magnetoimpedance ratio was increased by about $15 \%$ when a very thin layer of about $10 \mathrm{~nm}$ was deposited onto the wheel surface of the amorphous ribbon. Although the mechanism of the influence of the deposition of a thin magnetic layer onto the surface of the amorphous ribbon has to be clarified by additional study of the phenomenon, the proposed method may have the significant potential for technological applications for sensing of very small magnetic fields or when very well controlled anisotropy is necessary.

G.V. Kurlyandskaya thanks Spanish MCyT and Universidad de Oviedo for "Ramon Cajal" Felloship. Some images were taken at the shared SEM servise of the University of Ovedo.

\section{References}

[1] Beach R S and Berkowitz A E 1994 Appl. Phys. Lett. 64 3652

[2] Mohri K, Uchiyama T and Panina L 1997 Sensors Actuators A 591

[3] Vázquez M, Kurlyandskaya G V, García-Beneytez J M, Sinnecker J P, Barandiarán J M, Lukshina V A and Potapov A P 1999 IEEE Trans. Magn. 353358

[4] Sánchez M L, Prida V M, Hernando B, Kurlyandskaya G V, Santos J D, Tejedor M and Vázquez M 2002 Chin. Phys. Lett. 191870

[5] Xiao S Q, Liu Y H, Yan S S, Dai Y Y, Zhang L and Mei L M 2000 Phys. Rev. B 615734

[6] Nishibe Y, Yamadera H, Ohta N, Tsukada K and Nomomura Y 2000 Sensors and Actuators A 82155

[7] Dai Y Y, Xiao S Q, Liu Y H, Zhang L, Wu H and Zhang Y Z 2001 Chin. Phys. Lett. 18272

[8] Beach R S, Smith N, Platt F, Jeffers F and Berkowitz A E 1996 Appl. Phys. Lett. 682753

[9] Betancourt I, Valenzuela R and Vázquez M 2002 J. Appl. Phys 918423

[10] Iida S, Ishii O and Kambe S 1998 Jpn. J. Appl. Phys. 37 L869

[11] Amalou F and Gijs M A M 2002 Appl. Phys. Lett. 811654

[12] Panina L V and Mohri K 2000 Sensors Actuators 8171

[13] Kurlyandskaya G V, Yakabchuk H, Kisker E, Bebenin N G, García-Miquel H, Vázquez M and Vas'kovskiy V O $2001 \mathrm{~J}$. Appl. Phys. 906280

[14] Landau L D and Lifshitz E M 1975 Electrodynamics of Continuous Media (Oxford: Pergamon) p 195

[15] Kurlyandskaya G V, García-Miquel H, Vázquez M, Svalov A V and Vas'kovskiy V O 2002 J. Magn. Magn. Mater. 24935

[16] Tejedor M, Rubio H, Elbaile L and Iglesias R 1993 IEEE Trans. Magn. 293466

[17] Tejedor M, García J A, Carrizo J, Elbaile L and Santos J D 2002 J. Appl. Phys. 918435

[18] Kurlyandskaya G V, Sánchez M L, Hernando B, Gorria P, Prida V M and Tejedor M 2003 Appl. Phys. Lett. 823053

[19] Miyajima H and Sato K 1983 J. Appl. Phys. 474669

[20] Kurlyandskaya G V, Dmitrieva N V, Potapov A P, Lukshina V A, Voronova L M, Gervas'eva I V and Bebenin N G 1997 Phys. Met. Metallogr. 8341

[21] Hubert A and Schäfer R 1998 Magnetic Domains (Berlin: Springer) p 440

[22] Cerdeira A, Tejedor M and Fernandez A (at press) 